\title{
An Automated Approach towards Detection of Mitosis in Histopathological Images
}

\author{
Anand Raj Ulle \\ Department of Information science and Engineering \\ Sri Jayachamarajendra College of Engineering, Mysuru, India
}

\author{
T. N. Nagabhushan \\ Department of Information science and Engineering \\ Sri Jayachamarajendra College of Engineering, Mysuru, India
}

\author{
Nandini Manoli \\ Department of Pathology \\ JSS Medical College and Hospital, Mysuru, India
}

\begin{abstract}
Generally, the grade of a breast cancer is considered as an "aggressive potential" in the growth of a tumor. Breast cancer grading is characterized by three important factors, gland formation, nuclear pleomorphism, and mitosis count. In this research, an automated detection of mitosis from histopathological images is presented. From initial experiments, it has been observed that detection of mitosis becomes challenging, due to the similarity in size and shape compared to nonmitosis nuclei. Towards this end, several contributions have been made to automatically detect mitosis nuclei. From an Exhaustive experimentation, it is clear that mitotic texture shows discriminative features when compared to nonmitotic nuclei. To validate the performance of mitosis detection, two datasets from the MITOSIS-ATYPIA-14 challenge is considered. The proposed method is able to achieve $97 \%$ overall accuracy after feature reduction.
\end{abstract}

\section{Keywords}

Histopathological Images, Mitosis, Texture features, Patch extraction, Digital Pathology

\section{INTRODUCTION}

Breast cancer(BC) is the top one, a major cause of cancer in females, in India. Statistics show an increasing number of BC patients, especially in urban regions [18]. Also, the statistics show younger age female, most susceptible to BC. Therefore, early diagnosis would reveal prognostic factors that helps clinician plan suitable treatment. According to Nottingham grading system, pathologist grade tissue samples based on three important factors, (1) tubule formation, (2) nuclear pleomorphism, and (3) mitotic count. Amongst them, mitosis detection is an important feature in identifying the extent of tumor progression[1]. Traditionally, pathologists look for morphological changes in the nuclei and determine the mitotic count. This procedure is complicated and tedious for pathologists due to two reasons, (1) the size and shape of some of the mitotic nuclei are similar to nonmitotic nuclei, and (2) every day pathologist would see a massive amount of histopathological images. Clearly, there exist inter-observer variability leading to many false positives [29]. Over last decade, digital pathology has emerged as a promising field in the detection and progression of cancer from the histopathological images. Recently, there exist many research contributions towards accurate mitosis detection [14, 5, 21, 3, 27]. Accurate identification of mitotic nuclei poses a great challenge due to irregular size and shape of the nuclei, improper staining, and noise.

\section{COMPLEXITIES IN HISTOPATHOLOGICAL IMAGES}

It is evident from the literature on histopathological image analysis, that segmenting the nuclei is the fundamental step for an automated grading system. Nuclear segmentation poses a great challenge although there has been several attempts to solve it. Fig. 11 shows the complexities present in raw histopathological images. Extraction of nuclei from the histopathological images becomes challenging task since the size and shape of the nuclei are irregular, color intensities are non-homogenous, and texture variations across mitotic nuclei.
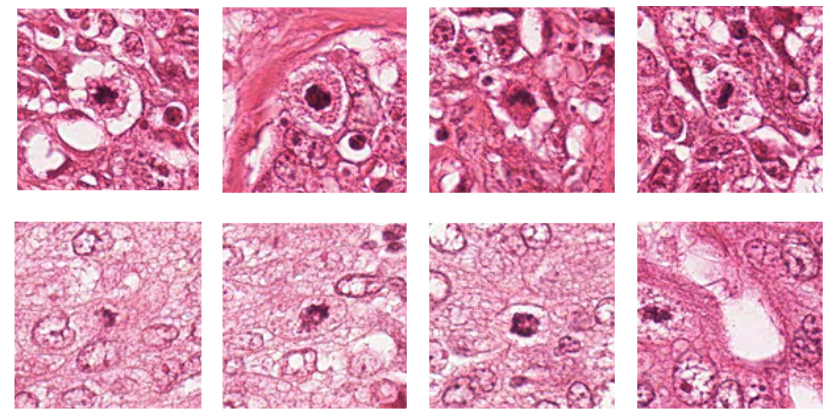

Fig. 1: Complexities in nuclei segmentation

\section{RELATED WORKS}

There exist several contributions towards nuclei detection and segmentation from H\&E stained histopathological images. Meftah et 
al [19] proposed spiking neural network for cell-microscopic detection. Kumar et al [16] proposed an adaptive eigenfilter based image segmentation for microscopic nuclei. It is clear from the literature that there exist few works on accurate detection of mitosis. Irshad et al [14] proposed blue-ratio image and threshold method for the detection of nuclei. Sommer et al [27] in their work, have used ilastik software [28] to segment mitotic nuclei and then applied CellCognition software [13] utilizing the global features of the cells such as shape and texture for candidate classification. Beevi et al [5] have used Localized Active Contour Model (LACM) to segment candidate nuclei. Sertel et al[26] proposed a pixel-level likelihood function and thresholding method for accurate detection of mitosis. Anari et al [4] proposed a fuzzy $\mathrm{C}$ means clustering to detect mitotic nuclei from immunohistochemistry (IHC) images of meningioma. Roullier et al[23] proposed a multi-resolution graph based method for automated detection of mitotic nuclei. Recently, FCM based nuclei segmentation[31] was proposed to detect and separate Leukemia nuclei. Marker-controlled watershed-based nuclei segmentation [30] for breast cancer tissue images were also proposed. Color deconvolution and morphological operators were used as preprocessing steps to remove irrelevant objects from the tissue images. Dong et al [7] proposed a method to discriminate benign from malignant in the intraductal proliferation of breast cancer. In their research work, authors have used Fiji [25] tool to segment the nuclei and then fine tuned using global thresholding approach.

\section{Observations}

From the literature, it is clear that identifying morphological changes in the histopathological images is utmost important for identifying mitosis. Some of the important findings from several studies suggest that texture analysis is also one of the important features in discriminating mitosis from nonmitosis. Also, on initial experiments with nuclei extraction, we found that the number of nonmitotic nuclei is higher compared to mitotic nuclei thus leading to imbalanced class. To address the above issues we propose a new method for nuclei segmentation based on extracting patches around the mitotic cell. Further, classification of mitotic nuclei is performed based on the extraction of texture, shape, and color features from these patches.

\section{PROPOSED METHOD}

Several methods [12, 22, 32, 6] have been proposed to identify nuclei present in histopathological images. From the outcomes of these research, it is evident that the existing methods perform well for regularly shaped nuclei but fail when the shape and size of the nucleus are varying. In the current research work, a simple approach to classifying mitosis nuclei is presented. Fig. 2 show the process of nuclei segmentation.

\subsection{Nuclei segmentation}

In order to extract mitotic nuclei, a patch of $120 \mathrm{x} 120$ pixels is used as bounding box to crop regions around the mitotic nuclei. Since the quality of image acquisition and staining procedure affect the segmentation accuracy, preprocessing of histopathological images, prior to segmentation, becomes inevitable. In the current research, the color normalization and color deconvolution [15] approaches are used to extract hematoxylin channel. Further, nuclei from these patches are segmented based on an Expectation-Maximization algorithm (EM) [8]. EM algorithm determines the posterior probabilities of each pixel $p$ representing one of the $\mathcal{C}$ classes, $\omega_{c}$ $=\{1,2, \ldots, C\}$, in a given image patch. In the current research work, the number of classes are considered to be $3, \omega_{c}=\{$ nuclei,stroma,background $\}$. Given an image patch the EM algorithm determines the posterior class probability densities, $\mathcal{P}\left(\omega_{c} \mid f(p)\right)$ given prior probability $\mathcal{P}\left(f(p) \mid \omega_{c}\right)$. The algorithm consists of two steps, the expectation, and the maximization step. In the expectation step the algorithm determines the prior Gaussian mixture parameters, $\eta=\mu, \Sigma, \mathcal{P}$. In the current research work, we have used $\mathrm{K}$-means algorithm to identify the prior Gaussian mixture parameters. In the maximization step, EM algorithm iteratively identifies the posterior class densities and update the Gaussian mixture parameters. At each iteration, the algorithm assigns pixels to class $\mathcal{C}$ that maximizes the posterior probabilities. In the current research work, the calculation of E-step and M-step are given below. Initialization step: In this step, the Gaussian prior parameters are estimated, $\eta$.

$$
\mathcal{P}\left(\omega_{c} \mid f(p)\right)=\frac{\mathcal{P}_{c} \mathcal{N}\left(f(p) \mid \mu_{c}, \Sigma_{c}\right)}{\Sigma_{i=1}^{C} \mathcal{P}_{i} \mathcal{N}\left(f(p) \mid \mu_{i}, \Sigma_{i}\right)}
$$

where,

$\mathcal{N}\left(f(p) \mid \mu_{c}, \Sigma_{c}\right)=(2 \pi)^{-} \frac{D}{2}\left|\Sigma_{c}^{0.5}\right| \exp \left\{\frac{1}{2}\left(f(p)-\mu_{c}^{i}\right)^{T} \Sigma_{c}^{-1}\left(f(p)-\mu_{c}^{i}\right)\right\}$

In the current research work, D is set to 3 , as the number of classes is 3

M-step: In this step, we update the Gaussian mixture parameters.

$$
\begin{gathered}
\mu_{c}^{i}=\frac{1}{n_{c}} \Sigma_{c=1}^{|C|} \mathcal{P}\left(D_{c} \mid f(p)\right) f(p) \\
\Sigma_{c}^{i}=\frac{1}{n_{c}} \Sigma_{c=1}^{|C|} \mathcal{P}\left(D_{c} \mid f(p)\right)\left(f(p)-\mu_{c}^{i}\right)\left(f(p)-\mu_{c}^{i}\right)^{T} \\
P_{c}^{i}=\frac{n_{c}}{|C|}
\end{gathered}
$$

After nuclei segmentation, the binary image contains unwanted regions (noise) that must be removed before further processing. Towards this end, Several well-established works on removing noise/artifacts from the histopathological images exist. However, after several experimentations on different histopathological images, it is found that the existing methods [7, 22| use fixed size as a threshold to remove smaller objects. Because the shape of the nuclei is not fixed, the existing methods fail to generalize on the shape. Therefore, a model is proposed in the current research to prune smaller objects as shown in Algorithm. 1.

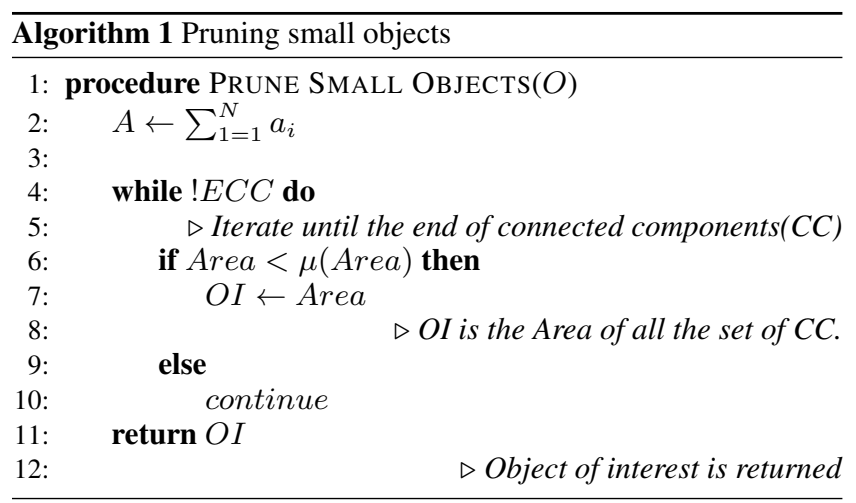




\section{Pre processing}

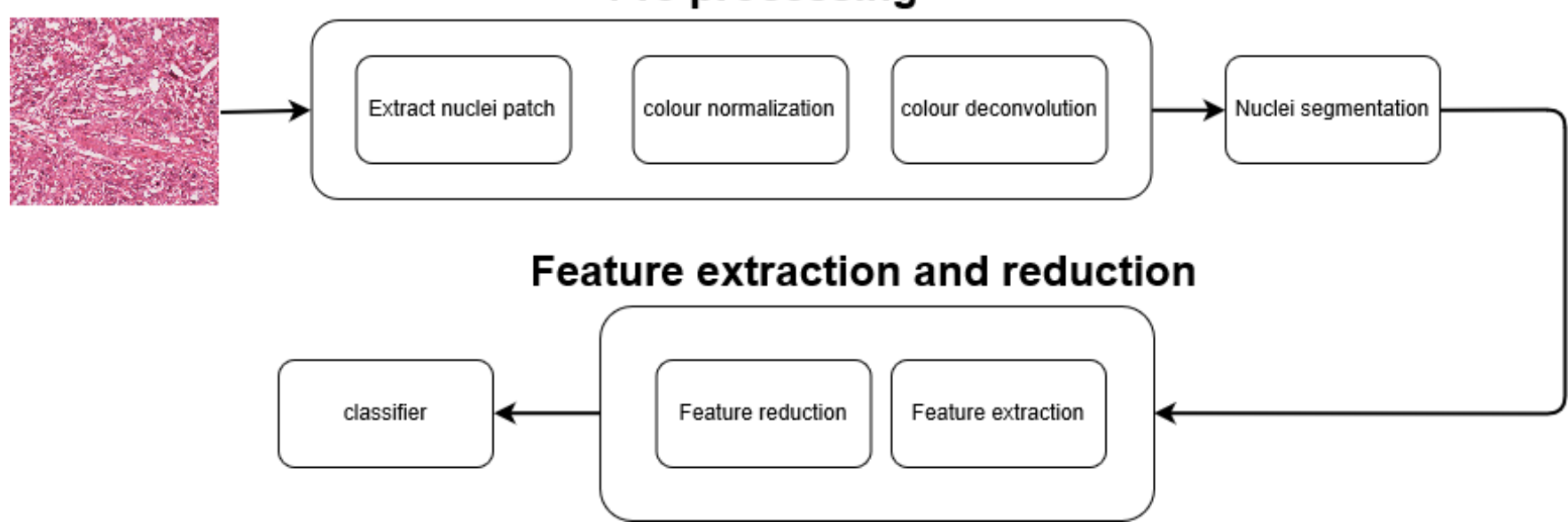

Fig. 2: The proposed approach for an automated segmentation and classification of mitotic nuclei from histopathological images

\subsection{Feature extraction}

Histopathological image analysis can be charecterized by three method. The first method is based on nuclei segmentation, that represents cellular changes, while the second method captures the texture properties, and the third method captures, the variations of color densities. The following features extracted in the current reearch work is shown in table 1 .

Table 1. : Hand-crafted Features

\begin{tabular}{|l||l|}
\hline Type & Features \\
\hline \hline Shape & Area, Perimeter, Solidity, circularity \\
\hline Texture & $\begin{array}{l}\text { Co-occurrence matrix, run-length matrix } \\
\text { Local phase quantization. }\end{array}$ \\
\hline Intensity & Histogram of color densities \\
\hline
\end{tabular}

\section{Shape features}

Nuclear features, are also one of the important features that describes mitotis behaviour. [17]. The following set of features are extracted based on the morphological changes in the cellular structure of each blob: Area, perimeter, solidity, and circularity. Finally, we extract 4D shape features that best describes the mitotis behaviour.

\section{Texture features}

Texture features are extracted from each detected nuclei. Three types of texture features are extracted, using co-occurrence matrix, run-length matrix, and local phase quantization.

Gray-Level-co-occurrence matrices Gray-level co-occurrence matrices(GLCM) are most commonly used method to extract texture features. In the current research work, eight adjacent directions are used to find GLCM, using this, 13 harlick features [10] are computed: Angular Second Moment, Contrast, Correlation, Variance,
Inverse Difference Moment, Sum Average, Sum Variance, Sum Entropy,Entropy, Difference Variance, Difference Entropy, Information Measure of Correlation I, Information Measure of Correlation II, Maximal Correlation Coefficient for all the eight directions. Finally, we obtain $13 \mathrm{D}$ feature vector by averaging across all eight directions.

Gray-Level-Run-Length matrices Adjacent pixel, with the same gray level constitue a run in a given direction $\mathrm{d}=\left\{0, \frac{\pi}{4}, \frac{\pi}{2}, \frac{3 \pi}{4}, \pi\right\}$. Run-length is a value that defines the number of time such a run occured. The gray-level run-length matrix (GRLM) defines a 2D matrix, where each element $x(i, j)$, in the matrix defines the run-length $\mathrm{j}$ for $i^{\text {th }}$ pixel, along the direction d [9]. In the current research work, the GRLM, is used to extract 10 features: Short run emphasis (SRE), long run emphasis (LRE), grey level non uniformity (GLN), run length non uniformity (RLN), low grey level runs emphasis (LGLRE), high grey level runs emphasis (HGLRE), short run low grey level emphasis (SRLGLE), short run high grey level emphasis (SRHGLE), long run low grey level emphasis (LRLGLE), and long run high grey level emphasis (LRHGLE). Finally, we obtain 10D feature vector by averaging across all eight directions.

Local phase quantization The Local Phase Quantization (LPQ) is a texture descriptor proposed by Ojansivu et al [20]. LPQ captures the blur invariance from the discrete Fourier transform. A window of $\mathrm{M} \times \mathrm{M}$ is used to extract the local phase information, using the 2D short-term Fourier transform (STFT). The quantized coefficients are represented as integer values ranging from 0-255 using binary coding described in []. A 256 bin histogram defines the values of phase information. For more details, refer to [20]. Finally, a 256D feature vector is obtained.

\section{Intensity features}

It is clearly evident from the Fig. 1, there exist intensity variations across the mitotic and nonmitotic nuclei. Usually, the mitotic nuclei take darker stains compared to nonmiotic nuclei [21]. Wang et al [33] in their work, have considered several intensity features: mean, median, variance, maximum/minimum ratio, range, interquartile range, kurtosis and skewness of patch intensities at seven color 
channels, for the classifying task. Recently, the color deconvolution [24] approach is used to extract hematoxylin channel. In the current research work, we identify the absorption capacity of hematoxylin stain at both miotic and nonmitotic nuclei using Eq 2. For rest of the mathematics, refer [11].

$$
A(\lambda)=-\ln \frac{I(\lambda)}{I_{0}(\lambda)}
$$

where $I(\lambda)=I_{0}(\lambda) . e^{-\delta(\lambda) \cdot c}, I_{0}(\lambda)$ is the spectral radiation intensity, $I(\lambda)$ is the transmitted spectral intensity, $\delta(\lambda)$ is the spectral molar optical density for a unified layer thickness and $\mathrm{c}$ is the dye concentration

\section{Classifiers}

Three different classifiers are used to measure the accuracy of mitotic detection: support vector machine (SVM), 5-nearest neighbour (5-NN), and TreeBagger(TB). An SVM is a type of classifier, that finds an optimal hyperplane that linearly separates all the features vectors, by projecting onto higher dimensional space. K-NN is a type of classifier that predicts the outcome of the unknown sample, by finding the distance between the unknown point and its nearest neighbour. In the current research work, we have used 5 nearest neighbours. From the literature, it is clear that single decision tree tends to overfit the data. Also, it is a well-established fact that classification based on TreeBagger provides better generalization even when there exit unbalanced classes. In our experimentations, we have considered 20 decision trees.

\section{DATA-SET DESCRIPTION}

To illustrate the methodology of nuclei extraction and classification, histopathological images from the Pathology Department at Pitiè-Salpêtrière Hospital in Paris, France [2] is considered. The slides are stained with standard hematoxylin and eosin (H\&E) dyes and they have been scanned by two slide scanners: Aperio Scanscope XT and Hamamatsu Nanozoomer 2.0-HT. In the current research work, all the images are taken from the Aperio Scanscope XT. This data-set consist of 1,136 frames at $40 \times$. The frames are RGB bitmap images in TIFF format. In the current research work, we have considered 2 dataset sample with different varying mitosis counts. Dataset 1 consists of 135 mitosis nuclei,and dataset 2 consists of 264 miotosis nuclei.

\section{RESULT}

In this section, we present the experimental analysis on segmentation and classification of histopathological images. Table 2 represents the quantitative results of classification for dataset 1 , while Table 3 show the classification accuracy for dataset 2 . Table 4 the performance evaluation of different classifier with feature reduction. The outcomes from this study show two important things: (1) classification of mitosis nuclei showed improvements after feature reduction, and (2) texture features played an important role in accurately classifying mitosis nuclei.

In this research, 224 histopathological images are considered for segmenting the nuclei. The dataset consists of 399 mitotic and 227 nonmitotic nuclei from the two datasets. To validate the nuclei segmentation process, expert pathologist were consulted and mitotic nuclei were marked. Also, the dataset itself had ground truth mitotic nuclei marked by two independent expert pathologists. The qualitative analysis of the segmentation algorithms is presented in Fig. 3

\subsection{Quantitative analysis of classifiers}

The performance of the classifier is measured based on different features extracted after nuclei segmentation. Table 2 Table 3 , and Table 4 show the comparative analysis of different classifiers without and with feature reduction respectively. The results show improvements in the weighted harmonic mean, from $97 \%$ to $98 \%$ after feature reduction. From the performance table, it is evident that the performance of SVM is better compared to other classifiers.

Table 2. : A performance measure of different classifiers for dataset 1 with different descriptors

\begin{tabular}{|c|c|c|c|c|c|c|}
\hline Descriptor & Classifier & Accuracy & F-Measure & PPV & Sensitivity & Specificity \\
\hline \multirow{3}{*}{ Shape } & SVM & 0.90 & 0.92 & 0.91 & 0.94 & 0.85 \\
\hline & KNN & 0.76 & 0.82 & 0.83 & 0.80 & 0.69 \\
\hline & $\mathrm{TB}$ & 0.70 & 0.76 & 0.75 & 0.77 & 0.58 \\
\hline \multirow{3}{*}{ GLCM } & SVM & 0.91 & 0.93 & 0.94 & 0.92 & 0.90 \\
\hline & KNN & 0.80 & 0.84 & 0.84 & 0.84 & 0.73 \\
\hline & $\mathrm{TB}$ & 0.79 & 0.83 & 0.82 & 0.84 & 0.70 \\
\hline \multirow{3}{*}{ Intensity } & SVM & 0.89 & 0.91 & 0.91 & 0.91 & 0.85 \\
\hline & KNN & 0.76 & 0.81 & 0.81 & 0.82 & 0.67 \\
\hline & $\mathrm{TB}$ & 0.79 & 0.83 & 0.83 & 0.84 & 0.71 \\
\hline \multirow{3}{*}{ Run-Length } & SVM & 0.90 & 0.92 & 0.90 & 0.94 & 0.84 \\
\hline & KNN & 0.76 & 0.79 & 0.74 & 0.86 & 0.64 \\
\hline & $\mathrm{TB}$ & 0.76 & 0.81 & 0.79 & 0.83 & 0.66 \\
\hline \multirow{3}{*}{ LPQ } & SVM & 0.96 & 0.96 & 0.95 & 0.98 & 0.93 \\
\hline & KNN & 0.64 & 0.71 & 0.70 & 0.72 & 0.53 \\
\hline & $\mathrm{TB}$ & 0.84 & 0.87 & 0.85 & 0.89 & 0.77 \\
\hline \multirow{3}{*}{ all features } & SVM & 0.95 & 0.95 & 0.94 & 0.97 & 0.92 \\
\hline & KNN & 0.67 & 0.73 & 0.71 & 0.75 & 0.55 \\
\hline & $\mathrm{TB}$ & 0.81 & 0.85 & 0.83 & 0.87 & 0.73 \\
\hline
\end{tabular}

SVM - Support vector machine, KNN - K nearest neighbor, TB - Tree Bagger

\section{CONCLUSION}

In this research, a simple approach to classify mitosis based on extracting different hand-crafted features is presented. Identifying mitotic changes in a given area of histopathological images is utmost important in predicting the progression of the breast tumor and hence, the present study assumes importance. Segmentation of mitotic nuclei poses a great challenge owing to irregular size, shape, and non-homogenous pixel intensity present in the nuclei. Even though there exists a well-focused research on nuclei segmentation by several researchers yet, they lack in accurately extracting the nuclei. In this research, several types of features are extracted from a segmented patch of 120x120 pixel and both quantitative and qualitative performance are evaluated. The method presented in this 
Table 3. : A performance measure of different classifiers for dataset 2 with different descriptors

\begin{tabular}{|c|c|c|c|c|c|c|}
\hline Descriptor & Classifier & Accuracy & F-Measure & PPV & Sensitivity & Specificity \\
\hline \multirow{3}{*}{ Shape } & SVM & 0.85 & 0.88 & 0.89 & 0.87 & 0.82 \\
\hline & $\mathrm{KNN}$ & 0.72 & 0.77 & 0.76 & 0.78 & 0.63 \\
\hline & ТВ & 0.72 & 0.77 & 0.78 & 0.77 & 0.64 \\
\hline \multirow{3}{*}{ GLCM } & SVM & 0.86 & 0.88 & 0.87 & 0.90 & 0.81 \\
\hline & $\mathrm{KNN}$ & 0.75 & 0.79 & 0.77 & 0.81 & 0.67 \\
\hline & TB & 0.75 & 0.79 & 0.78 & 0.81 & 0.67 \\
\hline \multirow{3}{*}{ Intensity } & SVM & 0.86 & 0.89 & 0.90 & 0.88 & 0.83 \\
\hline & KNN & 0.76 & 0.80 & 0.78 & 0.83 & 0.68 \\
\hline & TB & 0.73 & 0.77 & 0.76 & 0.79 & 0.64 \\
\hline \multirow{3}{*}{ Run-Length } & SVM & 0.84 & 0.87 & 0.87 & 0.88 & 0.80 \\
\hline & $\mathrm{KNN}$ & 0.71 & 0.76 & 0.76 & 0.76 & 0.62 \\
\hline & TB & 0.73 & 0.78 & 0.78 & 0.78 & 0.65 \\
\hline \multirow{3}{*}{ LPQ } & SVM & 0.96 & 0.97 & 0.97 & 0.97 & 0.95 \\
\hline & KNN & 0.81 & 0.84 & 0.83 & 0.86 & 0.74 \\
\hline & $\mathrm{TB}$ & 0.82 & 0.85 & 0.83 & 0.87 & 0.75 \\
\hline \multirow{3}{*}{ all features } & SVM & 0.94 & 0.95 & 0.95 & 0.96 & 0.93 \\
\hline & $\mathrm{KNN}$ & 0.84 & 0.87 & 0.86 & 0.88 & 0.79 \\
\hline & TB & 0.81 & 0.85 & 0.84 & 0.86 & 0.75 \\
\hline
\end{tabular}

Table 4. : A performance measure of different classifiers for dataset 1 and 2 with feature reduction

\begin{tabular}{lcccccc}
\hline Descriptor & Classifier & Accuracy & F-Measure & PPV & Sensitivity & Specificity \\
\hline & SVM & 0.97 & 0.97 & 0.97 & 0.98 & 0.95 \\
all features(dataset 1) & KNN & 0.78 & 0.83 & 0.83 & 0.82 & 0.71 \\
& TB & 0.84 & 0.87 & 0.85 & 0.90 & 0.77 \\
& SVM & 0.97 & 0.98 & 0.97 & 0.98 & 0.96 \\
all features(dataset 2) & KNN & 0.86 & 0.89 & 0.86 & 0.91 & 0.80 \\
& TB & 0.84 & 0.87 & 0.85 & 0.89 & 0.77 \\
\hline SVM - Support vector machine, KNN - K nearest neighbor, TB - Tree \\
\multicolumn{6}{c}{ Bagger }
\end{tabular}

research showed $97 \%$ accuracy in identifying mitosis nuclei. Further, the F-measure showed improved performance in nuclei classification after feature reduction.

\section{REFERENCES}

[1] Breast cancer pathology. http://pathology.jhu.edu/ breast/grade.php
[2] Mitosis-challenge-14 database. mitos-atypia-14.grand-challenge.org/

[3] Abdülkadir Albayrak and Gökhan Bilgin. Breast cancer mitosis detection in histopathological images with spatial feature extraction. In Sixth International Conference on Machine Vision (ICMV 2013), volume 9067, page 90670L. International Society for Optics and Photonics, 2013.

[4] Vahid Anari, Parvin Mahzouni, and Rasoul Amirfattahi. Computer-aided detection of proliferative cells and mitosis index in immunohistichemically images of meningioma. In Machine Vision and Image Processing (MVIP), 2010 6th Iranian, pages 1-5. IEEE, 2010.

[5] K Sabeena Beevi, Madhu S Nair, and GR Bindu. A multiclassifier system for automatic mitosis detection in breast histopathology images using deep belief networks. IEEE journal of translational engineering in health and medicine, 5:1-11, 2017.

[6] Ewert Bengtsson, C Wahlby, and Joakim Lindblad. Robust cell image segmentation methods. Pattern Recognition and Image Analysis C/c of Raspoznavaniye Obrazov i Analiz Izobrazhenii., 14(2):157-167, 2004.

[7] Fei Dong, Humayun Irshad, Eun-Yeong Oh, Melinda F Lerwill, Elena F Brachtel, Nicholas C Jones, Nicholas W Knoblauch, Laleh Montaser-Kouhsari, Nicole B Johnson, Luigi KF Rao, et al. Computational pathology to discriminate benign from malignant intraductal proliferations of the breast. PloS one, 9(12):e114885, 2014.

[8] H. Fatakdawala, J. Xu, A. Basavanhally, G. Bhanot, S. Ganesan, M. Feldman, J.E. Tomaszewski, and A Madabhushi. Expectationmaximization-driven geodesic active contour with overlap resolution (emagacor): Application to lymphocyte segmentation on breast cancer histopathology. Transactions on Biomedical Engineering, 2010.

[9] Mary M Galloway. Texture analysis using grey level run lengths. NASA STI/Recon Technical Report N, 75, 1974.

[10] Robert M Haralick, Karthikeyan Shanmugam, et al. Textural features for image classification. IEEE Transactions on systems, man, and cybernetics, (6):610-621, 1973.

[11] Peter Haub and Tobias Meckel. A model based survey of colour deconvolution in diagnostic brightfield microscopy: Error estimation and spectral consideration. Scientific reports, 5:12096, 2015.

[12] Lei He, L Rodney Long, Sameer Antani, and George R Thoma. Histology image analysis for carcinoma detection and grading. Computer methods and programs in biomedicine, 107(3):538-556, 2012.

[13] Michael Held, Michael HA Schmitz, Bernd Fischer, Thomas Walter, Beate Neumann, Michael H Olma, Matthias Peter, Jan Ellenberg, and Daniel W Gerlich. Cellcognition: timeresolved phenotype annotation in high-throughput live cell imaging. Nature methods, 7(9):747, 2010.

[14] Humayun Irshad, Sepehr Jalali, Ludovic Roux, Daniel Racoceanu, Lim Joo Hwee, Gilles Le Naour, and Frédérique Capron. Automated mitosis detection using texture, sift features and hmax biologically inspired approach. Journal of pathology informatics, 4(Suppl), 2013.

[15] Adnan Mujahid Khan, Nasir Rajpoot, Darren Treanor, and Derek Magee. A nonlinear mapping approach to stain normalization in digital histopathology images using image-specific color deconvolution. IEEE Transactions on Biomedical Engineering, 61(6):1729-1738, 2014. 
[16] Saravana Kumar, Sim Heng Ong, Surendra Ranganath, Fook Tim Chew, and Tan Ching Ong. Segmentation of microscope cell images via adaptive eigenfilters. In Image Processing, 2004. ICIP'04. 2004 International Conference on, volume 1, pages 135-138. IEEE, 2004.

[17] Christopher D Malon and Eric Cosatto. Classification of mitotic figures with convolutional neural networks and seeded blob features. Journal of pathology informatics, 4, 2013.

[18] Shreshtha Malvia, Sarangadhara Appalaraju Bagadi, Uma S Dubey, and Sunita Saxena. Epidemiology of breast cancer in indian women. Asia-Pacific Journal of Clinical Oncology, 2017.

[19] Boudjelal Meftah, Olivier Lezoray, Michel Lecluse, and Abdelkader Benyettou. Cell microscopic segmentation with spiking neuron networks. In International Conference on Artificial Neural Networks, pages 117-126. Springer, 2010.

[20] Ville Ojansivu and Janne Heikkilä. Blur insensitive texture classification using local phase quantization. In International conference on image and signal processing, pages 236-243. Springer, 2008.

[21] Angshuman Paul and Dipti Prasad Mukherjee. Mitosis detection for invasive breast cancer grading in histopathological images. IEEE Transactions on Image Processing, 24(11):4041-4054, 2015.

[22] Hady Ahmady Phoulady, Dmitry B Goldgof, Lawrence O Hall, and Peter R Mouton. Nucleus segmentation in histology images with hierarchical multilevel thresholding. In SPIE Medical Imaging, pages 979111-979111. International Society for Optics and Photonics, 2016.

[23] Vincent Roullier, Olivier Lézoray, Vinh-Thong Ta, and Abderrahim Elmoataz. Multi-resolution graph-based analysis of histopathological whole slide images: Application to mitotic cell extraction and visualization. Computerized Medical Imaging and Graphics, 35(7-8):603-615, 2011.

[24] Arnout C Ruifrok, Dennis A Johnston, et al. Quantification of histochemical staining by color deconvolution. Analytical and quantitative cytology and histology, 23(4):291-299, 2001.

[25] Johannes Schindelin, Ignacio Arganda-Carreras, Erwin Frise, Verena Kaynig, Mark Longair, Tobias Pietzsch, Stephan Preibisch, Curtis Rueden, Stephan Saalfeld, Benjamin Schmid, et al. Fiji: an open-source platform for biologicalimage analysis. Nature methods, 9(7):676, 2012.

[26] Olcay Sertel, Umit V Catalyurek, Hiroyuki Shimada, and Metin N Gurcan. Computer-aided prognosis of neuroblastoma: Detection of mitosis and karyorrhexis cells in digitized histological images. In Engineering in Medicine and Biology Society, 2009. EMBC 2009. Annual International Conference of the IEEE, pages 1433-1436. IEEE, 2009.

[27] Christoph Sommer, Luca Fiaschi, Fred A Hamprecht, and Daniel W Gerlich. Learning-based mitotic cell detection in histopathological images. In Pattern Recognition (ICPR), 2012 21st International Conference on, pages 2306-2309. IEEE, 2012.

[28] Christoph Sommer, Christoph Straehle, Ullrich Koethe, and Fred A Hamprecht. Ilastik: Interactive learning and segmentation toolkit. In Biomedical Imaging: From Nano to Macro, 2011 IEEE International Symposium on, pages 230-233. IEEE, 2011.

[29] Lisa A Teot, Richard Sposto, Anita Khayat, Stephen Qualman, Gregory Reaman, and David Parham. The problems and promise of central pathology review: development of a standardized procedure for the children's oncology group. Pediatric and Developmental Pathology, 10(3):199-207, 2007.

[30] M Veta, A Huisman, M.A. Viergever, van Diest, P.J., and J.P Pluim. Marker-controlled watershed segmentation of nuclei in h\&e stained breast cancer biopsy images. Biomedical Imaging, 2011.

[31] P. Viswanathan. Detection of leukemia based on morphological contour segmentation. Procedia Computer Science, 2015.

[32] Carolina Wählby, I-M SINTORN, Fredrik Erlandsson, Gunilla Borgefors, and Ewert Bengtsson. Combining intensity, edge and shape information for $2 \mathrm{~d}$ and $3 \mathrm{~d}$ segmentation of cell nuclei in tissue sections. Journal of microscopy, 215(1):6776, 2004.

[33] Haibo Wang, Angel Cruz Roa, Ajay N Basavanhally, Hannah L Gilmore, Natalie Shih, Mike Feldman, John Tomaszewski, Fabio Gonzalez, and Anant Madabhushi. Mitosis detection in breast cancer pathology images by combining handcrafted and convolutional neural network features. Journal of Medical Imaging, 1(3):034003, 2014. 

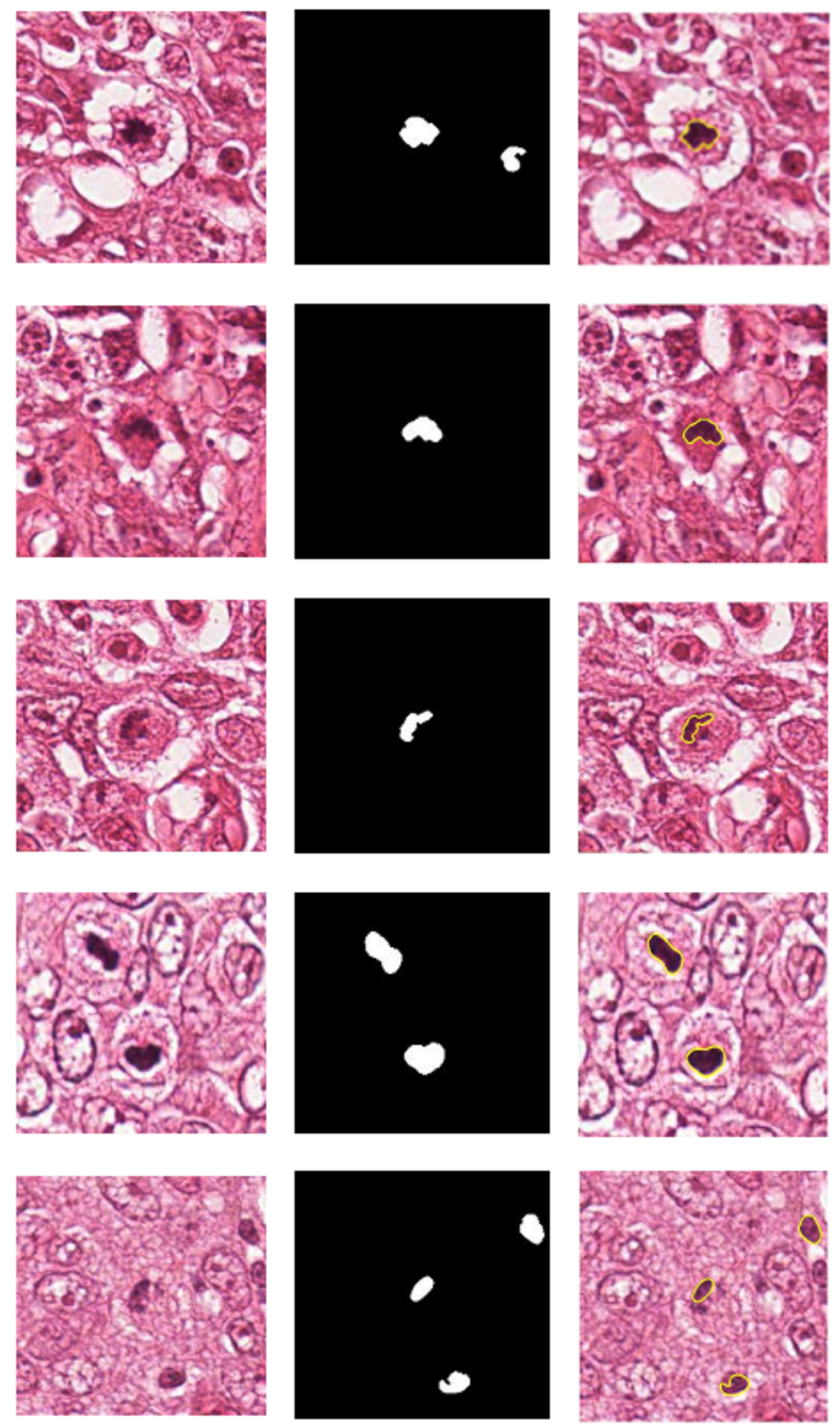

Fig. 3: Mitosis nuclei segmentation: column 1 represents patch extracted around the mitosis nuclei, column 2 represents the nuclei segmentation by the proposed method, and column 3 represents the segmented nuclei mapped on the original image. 